\title{
Influence of carbon nanotube length on toxicity to zebrafish embryos
}

\author{
This article was published in the following Dove Press journal: \\ International Journal of Nanomedicine \\ 18 July 2012 \\ Number of times this article has been viewed
}

\author{
Jinping Cheng',2 \\ Shuk Han Cheng' \\ 'Department of Biology and \\ Chemistry, City University of \\ Hong Kong, Hong Kong; ${ }^{2}$ State \\ Key Laboratory of Estuarine and \\ Coastal Research, East China Normal \\ University, Shanghai, China
}

\begin{abstract}
There is currently a large difference of opinion in nanotoxicology studies of nanomaterials. There is concern about why some studies have indicated that there is strong toxicity, while others have not. In this study, the length of carbon nanotubes greatly affected their toxicity in zebrafish embryos. Multiwalled carbon nanotubes (MWCNTs) were sonicated in a nitric acid solution for 24 hours and 48 hours. The modified MWCNTs were tested in early developing zebrafish embryo. MWCNTs prepared with the longer sonication time resulted in severe developmental toxicity; however, the shorter sonication time did not induce any obvious toxicity in the tested developing zebrafish embryos. The cellular and molecular changes of the affected zebrafish embryos were studied and the observed phenotypes scored. This study suggests that length plays an important role in the in vivo toxicity of functionalized CNTs. This study will help in furthering the understanding on current differences in toxicity studies of nanomaterials.
\end{abstract}

Keywords: length, carbon nanotubes, sonication, developmental toxicity, zebrafish

\section{Introduction}

While nanotechnology looms large with commercial promise and potential benefits, an equally large issue is the evaluation of the potential effects on human and environmental health. ${ }^{1}$ There is currently a difference of opinion in nanotoxicology studies of nanomaterials. There is concern regarding why some studies have indicated that there is strong toxicity, while others have not. ${ }^{2}$ Nanomaterials vary in terms of their chemical composition and physical dimensions. Carbon nanotubes (CNTs), one of the most promising nanomaterials, also vary in terms of their properties, depending on the manufacturing process. ${ }^{3}$ In addition, batch-to-batch variations within the same manufacturing facility as well as variability in the resultant product from different manufacturers exist. ${ }^{4}$ Small impurities associated with a nanomaterial or slight variations in structure/chemical composition may have a significant effect on biological response. ${ }^{5}$ These potential variations can affect assessments of expected toxicity.

There are differences in the results of toxicity studies on CNTs. ${ }^{6,7}$ The trouble is that in reality, each process of CNT manufacturing and modification produces various contaminants and inexact sizes of CNTs of interest. ${ }^{8-10}$ There is a growing consensus that characterization is an essential part of assessing the potential toxicity of nanomaterials in biological systems. ${ }^{11}$ Proper characterization of testing materials is important to ensure that results are reproducible, and also to provide the foundation for understanding the properties of nanomaterials that determine their biological effects. ${ }^{12}$

Raw CNTs tend to aggregate and agglomerate in an aqueous environment. Recent advances in functionalization, the attachment of functional groups on the sidewalls or 
terminals of the nanotubes, have led to aqueous dispersion and solubilization. ${ }^{13}$ Biological molecules, such as carbohydrates, nucleic acids, and proteins, as well as their precursors, are being used as functional groups to achieve dispersion in aqueous environments. ${ }^{14,15}$ These types of organic functionalization improve the dispersing abilities of CNTs, making them easier to interact with cells and become promising vehicles of delivery and biosensing. ${ }^{16}$ Coupled with their nanosize, they can biophysically or biochemically act with various subcellular or molecular structures once they are introduced inside a cell.

Since no general standards are available for testing samples, this is a concern, and sample characterization also varies. ${ }^{7}$ One report on water-dispersible single-wall CNTs showed that the cytotoxic response of cells in culture is dependent on the degree as well as mechanisms of functionalization. ${ }^{17}$ This article highlights the need to test out systematically production and functionalization processes for toxicity to achieve green chemistry of CNTs. Other studies have also highlighted the impact of surface characteristics on nanomaterial biocompatibility and point to the inadequacy of current size-dependent mechanistic paradigms, with nanoscale materials that lack unique or characteristic toxicity profiles. ${ }^{18}$ These findings strongly suggest the verification of biocompatibility data with the sample preparation process and the sample characterization data for each new class of materials or newly synthesized nanoparticles. Moreover, CNTs are frequently contaminated with metals, amorphous carbon, and other materials in the preparation process. ${ }^{19}$ Furthermore, the chemical modification and functionalization of CNTs requires much chemical processing, which can introduce functional groups with unwanted contaminants. ${ }^{14,15}$ Most toxicity studies therefore test for the toxic effects of the associated contaminants and not of the original materials. ${ }^{20}$ It is worthwhile to compare the observed toxicity with possible toxicity profiles generated in the preparation and functionalization of CNTs.

With the advanced development of nanomedicine, nanomaterial-incorporated products will potentially enter the human body, which makes in vivo toxicology profiles an important aspect in determining the safety of applications. The purpose of this study was to investigate the toxicity of CNTs with different lengths by using similar preparation processes. Zebrafish embryos were loaded with CNTs by microinjection at the one-cell stage. This study will help in furthering the understanding on the current differences in toxicity studies of nanomaterials. This study also highlights the importance of length information in the in vivo toxicology studies of nanomaterials.

\section{Materials and methods Preparation of modified CNTs}

Raw multiwalled (MW) CNTs were purchased from Nanostructured and Amorphous Materials (Houston, TX, USA). Purification, functionalization, and characterization were performed by using similar procedures as previously reported. ${ }^{21,22}$ Some MWCNTs were purified by refluxation in an aqueous nitric acid $\left(\mathrm{HNO}_{3}\right)$ solution $(2.6 \mathrm{M})$ for 48 hours (MWCNTs-48 h), and other CNTs were refluxed in the $\mathrm{HNO}_{3}$ solution $(2.6 \mathrm{M}$ ) for 24 hours (MWCNTs-24 h) as previously reported. After refluxation in an aqueous $\mathrm{HNO}_{3}$ solution, the resulting suspension was centrifuged $(\sim 1400 \mathrm{~g})$, and the sediment was collected and repeatedly washed with deionized water until a neutral $\mathrm{pH}$ was obtained. Purification procedures can remove carbonaceous impurities and metal catalysts. The purity of the sample was validated through thermogravimetric analysis (TGA) on a TGA/SDTA851e system (Mettler-Toledo, Columbus, $\mathrm{OH}$ ) with a typical heating rate of $10^{\circ} \mathrm{C} /$ minute. Bovine serum albumin (BSA)functionalized MWCNTs were prepared through amidation reactions. ${ }^{21}$ The reaction mixture was dialyzed against fresh deionized water for 3 days to obtain a fluorescein isothiocyanate (FITC)-labeled BSA-MWCNT sample. The commercially supplied FITC-BSA (Sigma-Aldrich, St Louis, MO) was used as the control. Electron microscopy imaging was conducted on a Tecnai 12 BioTWIN (Philips, Amsterdam, Netherlands) transmission electron microscope operated at $80 \mathrm{kV}$.

\section{Zebrafish maintenance and experimental setup}

Mature zebrafish were purchased from a local commercial source (Chong Hing Aquarium, Hong Kong). The zebrafish colony was maintained as previously described. ${ }^{5}$ Embryos were obtained by photo-induced spawning over green plants and cultured at $28.5^{\circ} \mathrm{C}$ in filtered tap water. The developmental stage of the embryos was measured according to hours postfertilization (hpf) and staged according to the method as described by Kimmel et al. ${ }^{23}$ After the light was turned on for photo-induced spawning, the embryos were collected within 10 minutes after fertilization and selected under a dissecting microscope for microinjection in experiments at the one-cell stage.

\section{Loading of modified CNTs into zebrafish embryos}

The samples were loaded into zebrafish embryos by microinjection at the one-cell stage as previously described. ${ }^{22}$ 
Briefly, the test samples were injected into the embryonic cells of zebrafish embryos with a nitrogen-driven microinjector (Narishige, Tokyo, Japan) within 30 minutes after fertilization. The microinjection pipette was calibrated every time before microinjection. The injection dosages were set with reference to the equivalent weight of the CNTs, and each experiment was repeated three to six times. The injected volume was approximately $2 \mathrm{~nL}$. The detailed amount of CNTs loaded into zebrafish embryos was listed in the result and determined by both the concentration of CNTs and the volume. The decreased dose of CNTs was achieved by diluting the samples to expected concentrations with phosphate-buffered saline (PBS). After microinjection, the embryos were incubated at $28.5^{\circ} \mathrm{C}$ for further development. The biodistribution of the CNTs was checked under a fluorescent microscope (Eclipse TE200; Nikon, Tokyo, Japan) as the modified CNTs were labeled with FITC. The affected phenotypes were scored and photographs of the defective embryos recorded with a light microscope (Eclipse TE200) at $80 \mathrm{hpf}$.

\section{Staining of cytoskeleton structure and nucleus of the treated embryos}

The staining of the microfilament structures and the nuclei of the zebrafish embryos was performed as described. ${ }^{24}$ For early stage embryos, a glass petri dish was used in the removal of chorion, and a $1 \%$ agarose-coated petri dish was used for staining. At the sphere stage (4 hpf), 50\% epiboly, and 75\% epiboly, the embryos were fixed with $4 \%$ paraformaldehyde PBS overnight at $4^{\circ} \mathrm{C}$, after which they were thoroughly washed with PBS. All subsequent washing steps were performed for 5 minutes and carried out with gentle shaking. To label the F-actin and nucleus, embryos were incubated with $0.08 \mu \mathrm{g} / \mathrm{mL}$ rhodamine phalloidin (Invitrogen, Carlsbad, CA) and Sytox Green (Invitrogen), at room temperature (RT) for 1 hour in the dark, after which they were extensively washed with PBS with $0.1 \%$ Tween 20 and $1 \%$ dimethyl sulfoxide, which contained 1\% BSA. Images were collected through water immersion on Achroplan $\times 20 / 0.5 \mathrm{NA}$ and $\times 40 / 0.75$ NA objectives on a C1 confocal microscopy system (Nikon). The FITC and rhodamine fluorescence were visualized by using 488-nm excitation/520-nm emission and 568-nm excitation/585-nm emission, respectively.

\section{TEM observation of embryonic cells in affected embryos}

Embryonic cells loaded with modified CNTs were studied by transmission electron microscopy (TEM) and compared with the untreated control. Zebrafish embryos loaded with BSA-MWCNTs were collected and fixed in a fixation buffer (2\% paraformaldehyde, $2.5 \%$ Gluta, $0.1 \mathrm{M}$ cacodylate buffer, $\mathrm{pH} 7.2,4 \%$ sucrose, $1 \%$ tannic acid) overnight at $4^{\circ} \mathrm{C}$. The fixed embryos were washed and then postfixed with $1 \%$ osmium tetroxide in $0.1 \mathrm{M}$ cacodylate buffer ( $\mathrm{pH}$ 7.2) at RT for 2 hours. The postfixed embryos were washed and then gradually dehydrated with ethanol. The dehydrated embryos were transferred into acetone before being transferred into resin. The embryos were embedded in pure resin for 4 hours at RT, and then the resin was polymerized at $70^{\circ} \mathrm{C}$ for 2 days before sectioning. The embryos were sectioned $(70 \mathrm{~nm})$ by using an Ultracut UCT (Leica, Solms, Germany) and placed on copper grids and double stained with a 5\% uranyl acetate solution and Reynolds lead citrate solution. The tissue was examined and photographed with a transmission electron microscope (Tecnai 12 BioTWIN), which was operated at $80 \mathrm{kV}$.

\section{Results and discussion}

\section{Sample characterization}

The prepared CNTs, after purification, were studied by TGA and characterized by TEM. After purification, the MWCNTs were analyzed by TGA, which indicated that after purification, the MWCNTs comprised $98 \%$ of the total sample weight. ${ }^{22}$ As shown in Figure 1, both BSA-modified CNTs disperse well after a successful functionalization. The average diameter of the prepared BSA-MWCNTs is about $19.9 \pm 8.25 \mathrm{~nm}$, and median diameter $17.5 \mathrm{~nm}$. Both the TGA and TEM characterization data show that the purified MWCNT samples are largely free from metal particles. It was observed that the sonication time can greatly affect the length of the purified MWCNTs. When MWCNTs were sonicated in an $\mathrm{HNO}_{3}$ solution for 24 hours, the average length of the prepared BSA-MWCNTs (MWCNTs-24 h) was about $0.8 \pm 0.5 \mu \mathrm{m}$, and median length $0.7 \mu \mathrm{m}$. When the MWCNTs were sonicated in an $\mathrm{HNO}_{3}$ solution for 48 hours (MWCNTs-48 h), the average length of the prepared BSAMWCNTs (MWCNTs-24 h) was about $0.2 \pm 0.1 \mu \mathrm{m}$, and median length $0.17 \mu \mathrm{m}$. MWCNTs-48 h (Figure 1A) are much shorter as compared with MWCNTs-24 h (Figure 1B), and this can be explained by the doubled length of sonication time in $\mathrm{HNO}_{3}$ during purification.

\section{Failure of epiboly initiation in affected embryos}

We have previously reported that successfully purified and functionalized MWCNTs had relatively good biocompatibility 

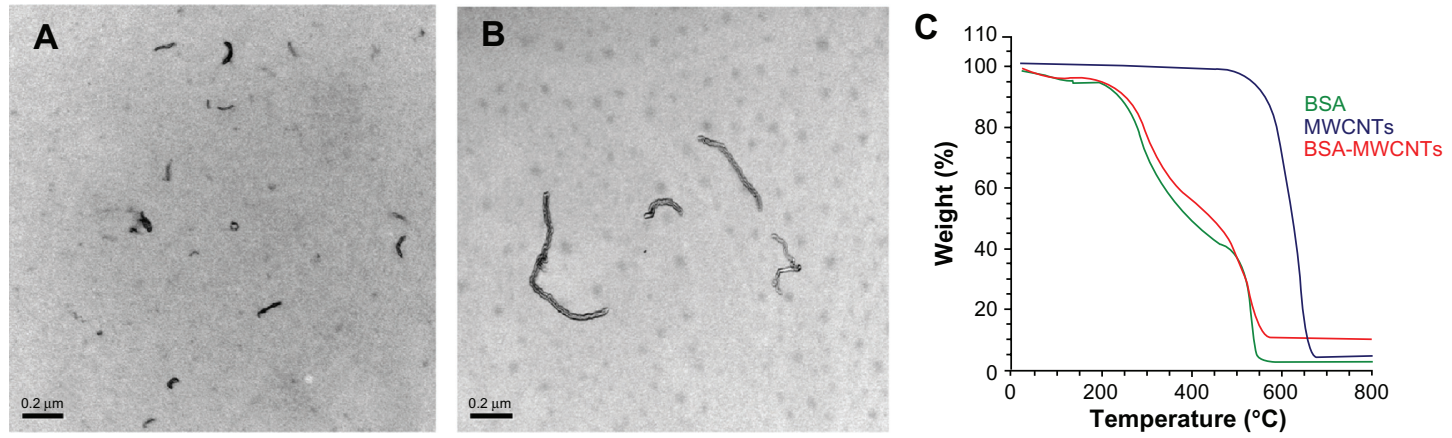

Figure I Representative transmission electron microscopy images of MWCNTs-48 h (A) and MWCNTs-24 h (B). C is the TGA analysis data of BSA-MWCNTs. Note: Scale bars, $200 \mathrm{~nm}$. MWCNTs-48 h refers to BSA-MWCNTs that had been purified by refluxation in an aqueous nitric acid (HNO3) solution (2.6 M) for 48 hours. MWCNTs-24 h refers to BSA-MWCNTs that had been purified by refluxation in an aqueous nitric acid (HNO3) solution (2.6 M) for 24 hours.

Abbreviations: BSA-MWCNT, bovine serum albumin-functionalized multiwalled carbon nanotube; TGA, thermogravimetric analysis.

in zebrafish embryos ${ }^{22}$ and mammalian cells. ${ }^{25}$ It was found that shorter length of MWCNTs-48 $\mathrm{h}$ induced severe toxicity in zebrafish embryos when injected at the one-cell stage, while the FITC-BSA control showed good biocompatibility. Previous studies have reported that long and rigid MWCNTs are much more toxic than functionalized MWCNTs, ${ }^{26}$ and longer raw MWCNTs cause inflammation and granulomas in normal mice by interacting with mesothelium. ${ }^{27}$ This study suggests that shorter BSA-MWCNTs are more toxic in zebrafish embryos after injection.

The embryos loaded with FITC-BSA developed normally as untreated controls. These embryos reached the shield stage at $6 \mathrm{hpf}$ (Figure $2 \mathrm{~A}$ and B), and 75\% epiboly at $8 \mathrm{hpf}$ (Figure 2E and F). The concentration of the MWCNTs (CNTs equivalent weight) was determined using the TGA method, and the stock concentration of FITC-BSA-MWCNTs was about $1 \mathrm{mg} / \mathrm{mL}$ (equivalent weight of CNTs). The embryos injected with 2 ng (equivalent weight of CNTs) of FITClabeled BSA-MWCNTs (MWCNTs-48 h) exhibited adverse toxic effects. These affected embryos were developmentally arrested until death at 8-10 hpf (Figure 2C and G). Epiboly did not initiate in these embryos. The fluorescent images showed that the BSA-MWCNTs are evenly distributed among the blastoderm cells and do not enter the yolk cell during epiboly arrest (white arrows in Figure 2D and H).

\section{Malformation phenotypes with decreased dosage}

When the dosage of loaded BSA-MWCNTs (MWCNTs-48 h) was less than $5 \mathrm{pg}$ (equivalent weight of CNTs), the treated
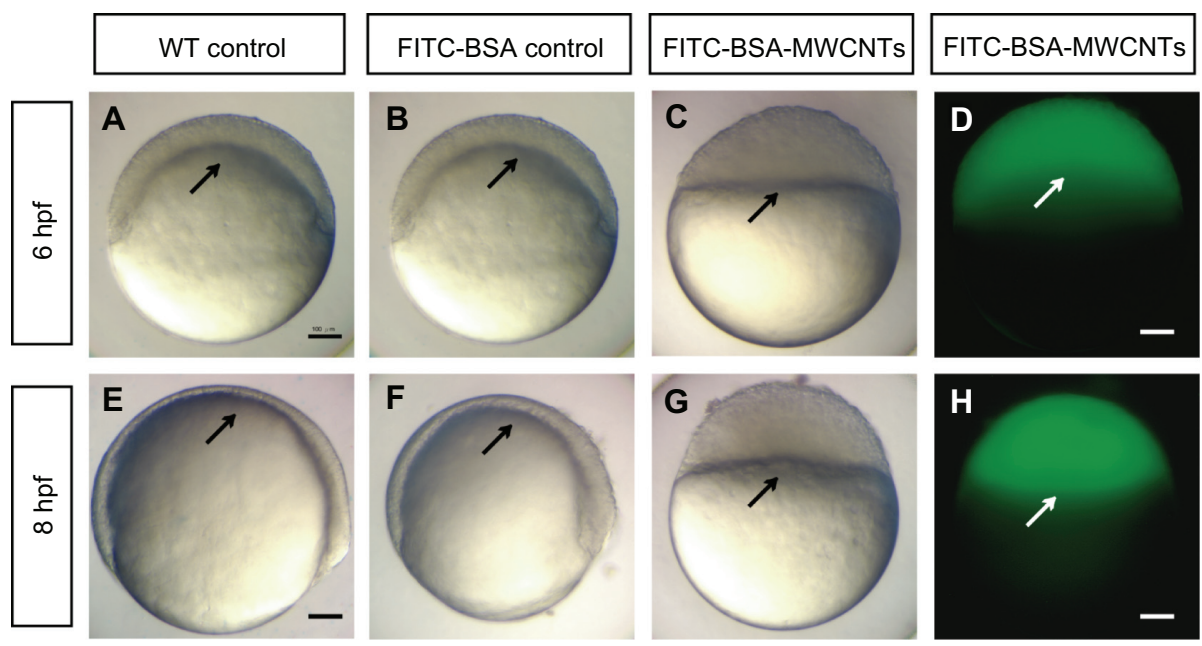

Figure 2 (A-H) Embryos loaded with short-length BSA-MWCNTs (MWCNTs-48 h) unable to initiate epiboly $(\mathrm{n}=300)$. Light microscope images of wild-type control embryos at $6 \mathrm{hpf}(\mathbf{A})$ and $8 \mathrm{hpf}(\mathbf{E})$. Light microscope images of embryos loaded with FITC-BSA (BSA equivalent weight) at 6 hpf (B) and 8 hpf (F). Light microscope images of embryos loaded with $2 \mathrm{ng}$ of FITC-BSA-MWCNTs at $6 \mathrm{hpf}(\mathbf{C})$ and $8 \mathrm{hpf}(\mathbf{G})$. Fluorescent microscope images of embryos loaded with 2 ng of FITC-BSA-MWCNTs (MWCNTs-48 h) at $6 \mathrm{hpf}(\mathbf{D})$ and $8 \mathrm{hpf}(\mathbf{H})$.

Note: Scale bar, $250 \mu \mathrm{m}$.

Abbreviations: FITC-BSA-MWCNT, fluorescein isothiocyanate-labeled bovine serum albumin-functionalized multiwalled carbon nanotube; hpf, hours postfertilization; WT, wild type. 
embryos passed the epiboly stage and went on to further embryonic development. The malformation percentages are $36.4 \%$ and $27.9 \%$ for embryos loaded with $4 \mathrm{pg}$ and $0.4 \mathrm{pg}$ of BSA-MWCNTs (MWCNTs-48 h) (equivalent weight of CNTs), respectively. The affected embryos have severe edema, distorted notochords, dissociated muscles, flattened midbrain-hindbrain boundaries, small eyes, delayed yolk sac and yolk extension resorption, and shortened body length, as shown in Figure 3. The defective embryos also have impaired swimming patterns. Endogenous alkaline phosphatase staining illustrated that the embryos with severe edema also have disorganized blood vessels and missing vascular endothelial cells (data not shown). However, affected embryos with other defective phenotypes have proper blood vessel systems (data not shown). The cardiovascular system is not the only component that the shorter BSA-MWCNTs (MWCNTs-48 h) attacked. Most of the affected embryos have more than one type of defect, and this might indicate that the influence is multiorgan targeted.

\section{Cytoskeleton structure and cell movement in the affected embryos}

Double staining of F-actin and Sytox Green was performed to mark the cell morphologies and nuclei of the affected embryos (Figure 4). The wild-type control embryos showed well-organized cells at the sphere and epiboly stages (yellow arrowheads in Figure 4A-C). The wild-type controls generated continuous punctate bands of actin at the leading edge of the enveloping layer (yellow arrow in Figure 4C) at 75\% epiboly. The embryos loaded with shorter BSA-MWCNTs (MWCNTs-48 h) exhibited gradual loss of nuclei by Sytox Green staining in the blastoderm and yolk syncytial layer as well as loss of the punctuate actin band typically found in embryos that reach 75\% epiboly (Figure 4D-F). The affected embryos displayed developmental arrest and maintained an appearance that is usually found at the sphere stage in the later developmental stages (Figure 2C and G; data not shown). In the affected embryos, some nuclei are irregular or even missing (white arrowhead in Figure 4D) at $4 \mathrm{hpf}$, and the nuclei in the yolk syncytial layer are missing from $4 \mathrm{hpf}$ to $50 \%$ epiboly (the region within the white square dots in Figure 4D and E), and more nuclei are lost at 75\% epiboly (white arrowheads in Figure 4F). The punctuate actin band is not detected at $8 \mathrm{hpf}$ (white arrow in Figure 4F).

Irregular nuclei are also found in the embryos at other developmental stages both by using Sytox Orange staining (Figure 5) and TEM observation (Supplementary Figure 1). In the embryos loaded with shorter-length BSA-MWCNTs
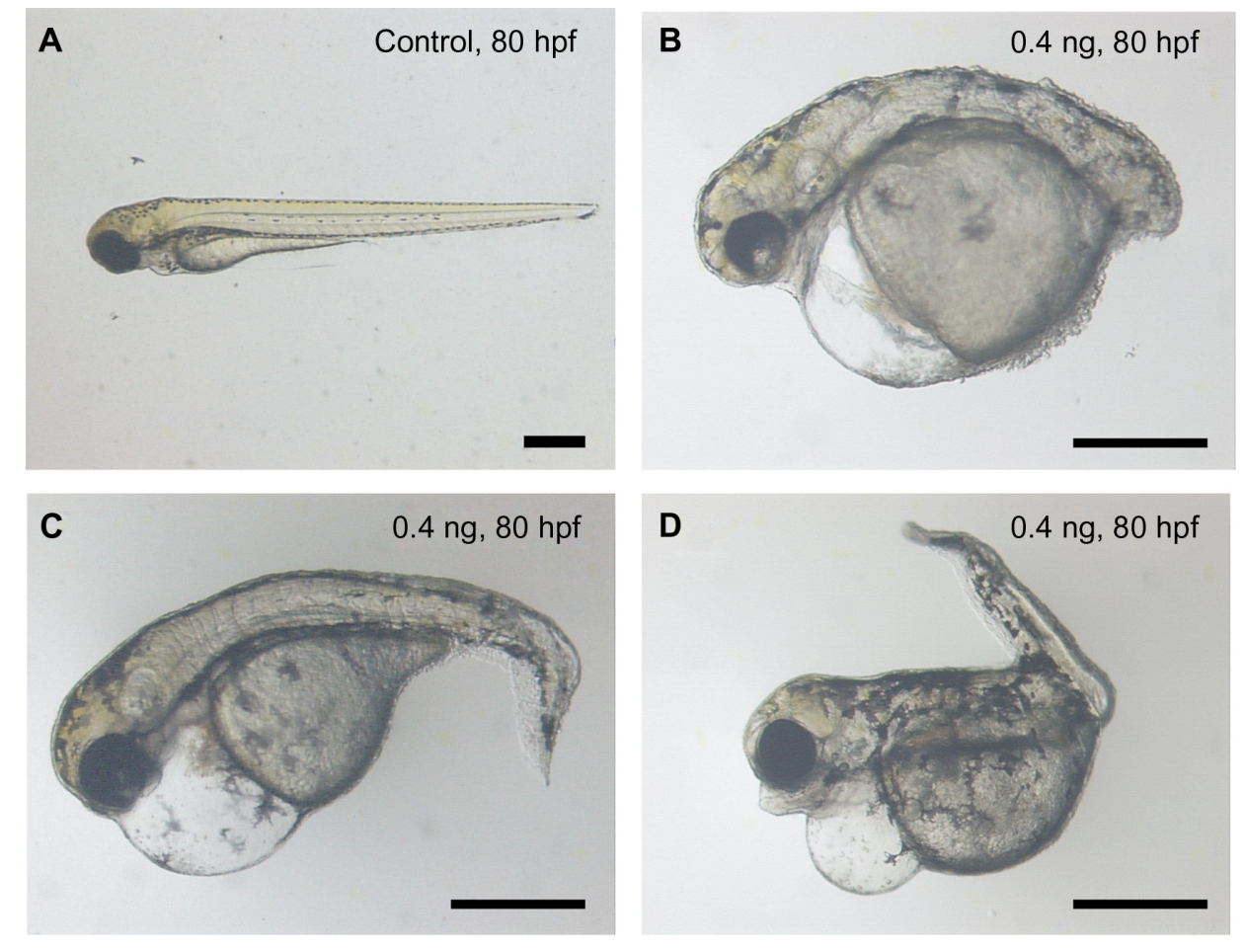

Figure 3 Representative images of control (A) and treated embryos (B, C and D) at 80 hpf. The malformaed embryos were loaded with a shorter length of BSA-MWCNTs (MWCNTs-48 h) (0.4 ng) at I-cell stage. The affected embryos had severe edema, distorted notochords, dissociated muscles, flattened midbrain-hindbrain boundaries, small eyes, delayed yolk sac and yolk extension resorption, and shortened body length, as shown in B, C and D.

Note: Scale bar, $500 \mu \mathrm{m}$. MWCNTs-48 h refers to BSA-MWCNTs that had been purified by refluxation in an aqueous nitric acid (HNO3) solution (2.6 M) for 48 hours.

Abbreviation: BSA-MWCNT, bovine serum albumin-functionalized multiwalled carbon nanotube. 


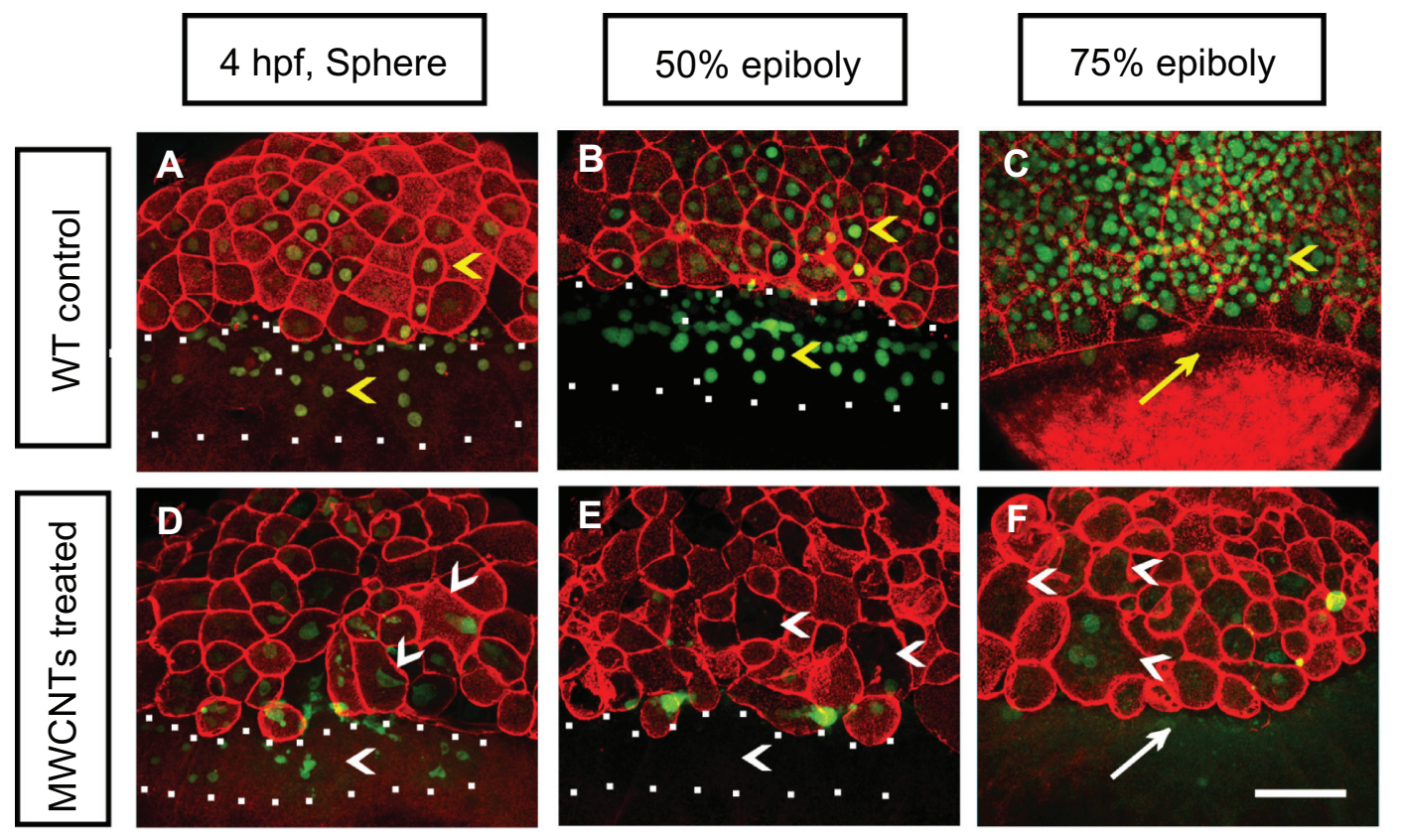

Figure 4 (A-F) Nuclei and morphologies of embryonic cells in affected embryos. Each image is a projected stack of confocal optical sections. Representative images of control (A-C) and embryos loaded with 2 ng of BSA-MWCNTs (MWCNTs-48 h) at 4 hpf (sphere stage) (D), 50\% epiboly (E), and 75\% epiboly (F).

Note: $\mathrm{n}=10$; scale bar, $50 \mu \mathrm{m}$.

Abbreviations: BSA-MWCNT, bovine serum albumin-functionalized multiwalled carbon nanotube; hpf, hours postfertilization; WT, wild type.

(MWCNTs-48 h), some of the nuclei of the blastoderm cells are lost or have irregular shapes at $2.5 \mathrm{hpf}$ (red arrows in Figure 5B) as compared with the control (Figure 5A). More and more nuclei are lost or have irregular shapes at $4 \mathrm{hpf}$ (red arrows in Figure 5D). The embryos loaded with FITC-BSA
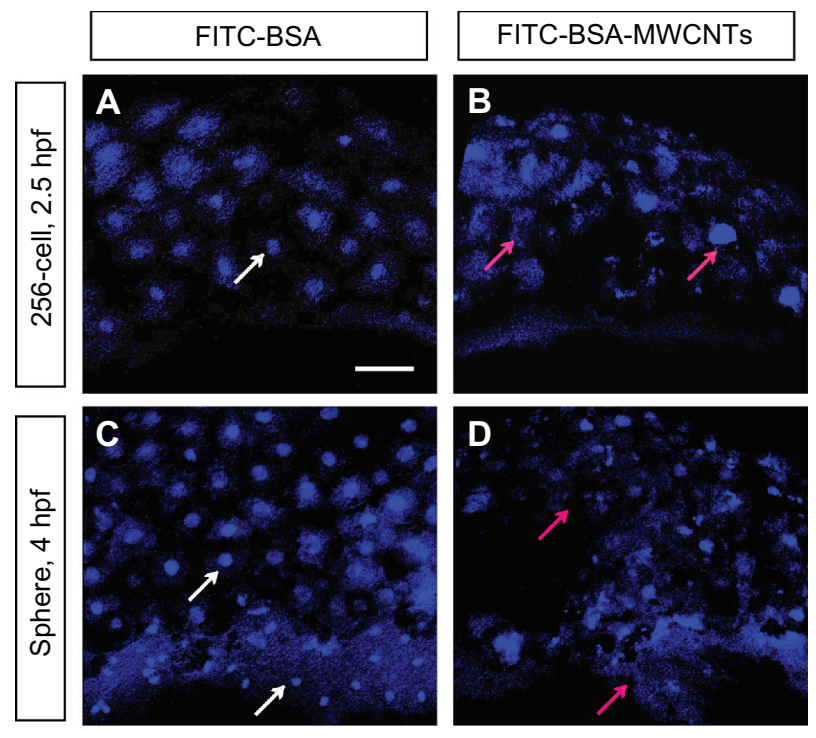

Figure 5 (A-D) The nuclei of blastoderm cells at $2.5 \mathrm{hpf}$ and $4 \mathrm{hpf}$ with Sytox Orange nucleus staining $(n=10)$. Embryos loaded with FITC-BSA $(\mathbf{A}$ and $\mathbf{C})$ and $2 \mathrm{ng}$ of BSA-MWCNTs (MWCNTs-48 h) (B and D) at I-cell stage, respectively. Note: Scale bar, $50 \mu \mathrm{m}$.

Abbreviations: FITC-BSA-MWCNT, fluorescein isothiocyanate-labeled bovine serum albumin-functionalized multiwalled carbon nanotube; hpf, hours postfertilization. are not affected, and the nuclei of the blastoderm cells are well organized and have regular round shapes (white arrows in Figure 5A and C).

The nucleus staining results were reidentified under a transmission electron microscope with ultrathin sections of the affected embryos. The microscope images showed that the blastoderm cell nuclei of the affected embryos have an irregular shape, and some micronuclei also appear at $6 \mathrm{hpf}$ (Supplementary Figure 1). The cells in the untreated embryos have regular round or horseshoe-shaped nuclei at $6 \mathrm{hpf}$ (Supplementary Figure 1F).

\section{Subcellular morphology change}

The morphology changes of the embryonic cells and the response of the internal organelles inside the embryonic cells after the loading of the materials were also investigated. TEM observation of the blastoderm cells of the affected embryos (Figure 6) demonstrated that the membranes of the enveloping cells are disrupted, and the deep-layer cells detach and have irregular nuclei with condensed chromatins. The morphology changes of the embryonic cells, especially the deep layer cells, suggest an apoptosis process. It is known that the characteristic breakdown of nuclei during apoptosis comprises collapse and fragmentation of the chromatins, degradation of the nuclear envelopes, and nuclear blebbing, which result in the formation of micronuclei. 

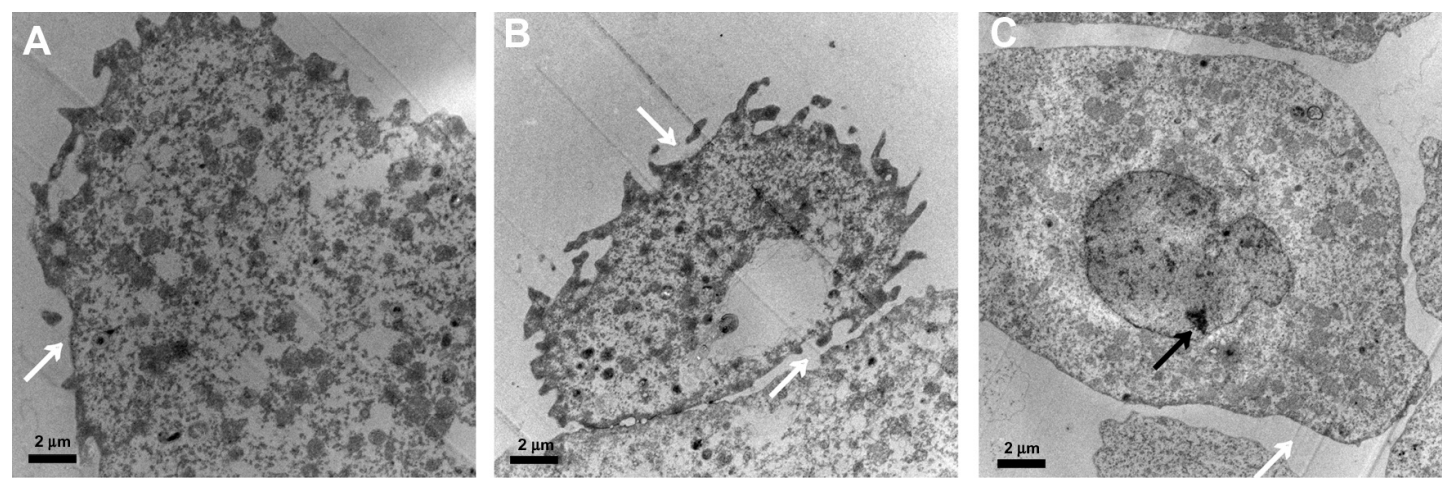

Figure 6 Transmission electron microscope images of the blastoderm cells of affected embryos loaded with BSA-MWCNTs (MWCNTs-48 h) at 6 hpf. The membranes of the enveloping cells were disrupted (A and $\mathbf{B})$. The deep-layer cells detached and had irregular nuclei with condensed chromatins (C).

Note: The white arrow points to corrupted membrane, and black arrow to irregular nucleus. Scale bar, $2 \mu \mathrm{m}$.

Abbreviations: BSA-MWCNT, bovine serum albumin-functionalized multiwalled carbon nanotube; hpf, hours postfertilization.

\section{Conclusion}

Given the various promises and potentials of nanotechnology and its applications, evaluation of the potential hazards related to exposure to nanomaterials and their products has become an emerging area in toxicology and health risk assessment. ${ }^{28}$ The novel properties of engineered nanomaterials may lead to the potential for different toxicities compared with bulk material. ${ }^{29}$ Nanotoxicology is thus emerging as an important subdiscipline of nanotechnology. ${ }^{6}$ The study of the interaction between nanostructures with biological systems emphasizes much on the relationship between the physical and chemical properties of nanostructures with induction of toxic biological responses. ${ }^{30}$ When it comes to in vivo testing systems, the interactions of nanostructures with biological components, such as proteins and cells, are extremely complicated. ${ }^{31,32}$ When a certain toxicity profile of testing nanomaterials is observed, care should be taken with specific parameters of the tested samples. A small change in one step can always make a big difference in the bioproperties of the prepared nanomaterial samples. The present study highlights the importance of sample characterization and suggests that length plays an important role in the in vivo toxicity of functionalized carbon nanotubes.

\section{Acknowledgments}

The work described in this paper was substantially supported by a grant from the National Natural Science Foundation of China to JP Cheng (grant no 41101489). Prof Ya-ping Sun's lab and Dr Wing Lin Poon are thanked for experimental assistance.

\section{Disclosure}

The authors report no conflicts of interest in this work.

\section{References}

1. Holsapple MP, Lehman-McKeeman LD. Forum series: research strategies for safety evaluation of nanomaterials. Toxicol Sci. 2005; $87: 315$.

2. Monteiro-Riviere NA, Inman AO. Challenges for assessing carbon nanomaterial toxicity to the skin. Carbon. 2006;44:1070-1078.

3. Baughman RH, Zakhidov AA, de Heer WA. Carbon nanotubes - the route toward applications. Science. 2002;297:787-792.

4. Valsami-Jones E, Berhanu D, Dybowska A, et al. Nanomaterial synthesis and characterization for toxicological studies: $\mathrm{TiO} 2$ case study. Mineral Mag. 2008;72:515-519.

5. Cheng J, Flahaut E, Cheng SH. Effect of carbon nanotubes on developing zebrafish (Danio rerio) embryos. Environ Toxicol Chem. 2007;26:708-716.

6. Donaldson K, Aitken R, Tran L, et al. Carbon nanotubes: a review of their properties in relation to pulmonary toxicology and workplace safety. Toxicol Sci. 2006;92:5-22.

7. Dumortier H, Lacotte S, Pastorin G, et al. Functionalized carbon nanotubes are non-cytotoxic and preserve the functionality of primary immune cells. Nano Lett. 2006;6:1522-1528.

8. Ebbesen TW, Ajayan PM. Large-scale synthesis of carbon nanotubes. Nature. 1992;358:220-222.

9. Cassell AM, Raymakers JA, Kong J, Dai HJ. Large scale CVD synthesis of single-walled carbon nanotubes. J Phys Chem B. 1999; 103:6484-6492.

10. Shi ZJ, Lian YF, Liao FH, et al. Large scale synthesis of single-wall carbon nanotubes by arc-discharge method. J Phys Chem Solids. 2000;61:1031-1036.

11. Hood E. Nanotechnology: looking as we leap. Environ Health Perspect. 2004;112:A740-A749.

12. Powers KW, Brown SC, Krishna VB, Wasdo SC, Moudgil BM, Roberts SM. Research strategies for safety evaluation of nanomaterials. Part VI. Characterization of nanoscale particles for toxicological evaluation. Toxicol Sci. 2006;90:296-303.

13. Fu KF, Sun YP. Dispersion and solubilization of carbon nanotubes. J Nanosci Nanotechnol. 2003;3:351-364.

14. Lin Y, Taylor S, Li HP, et al. Advances toward bioapplications of carbon nanotubes. J Mater Chem. 2004;14:527-541.

15. Sinha N, Yeow JTW. Carbon nanotubes for biomedical applications. IEEE Trans Nanobioscience. 2005:4:180-195.

16. Bianco A, Kostarelos K, Partidos CD, Prato M. Biomedical applications of functionalised carbon nanotubes. Chem Commun. 2005:571-577.

17. Sayes CM, Liang F, Hudson JL, et al. Functionalization density dependence of single-walled carbon nanotubes cytotoxicity in vitro. Toxicol Lett. 2006;161:135-142.

18. Stern ST, McNeil SE. Nanotechnology safety concerns revisited. Toxicol Sci. 2008;101:4-21. 
19. Inami N, Mohamed MA, Shikoh E, Fujiwara A. Synthesis-condition dependence of carbon nanotube growth by alcohol catalytic chemical vapor deposition method. Sci Technol Adv Mater. 2007;8:292-295.

20. Worle-Knirsch JM, Pulskamp K, Krug HF. Oops they did it again! Carbon nanotubes hoax scientists in viability assays. Nano Lett. 2006;6:1261-1268.

21. Huang WJ, Taylor S, Fu KF et al. Attaching proteins to carbon nanotubes via diimide-activated amidation. Nano Lett. 2002;2:311-314.

22. Cheng JP, Chan CM, Veca LM, et al. Acute and long-term effects after single loading of functionalized multi-walled carbon nanotubes into zebrafish (Danio rerio). Toxicol Appl Pharmacol. 2009;235:216-225.

23. Kimmel CB, Ballard WW, Kimmel SR, Ullmann B, Schilling TF. Stages of embryonic development of the zebrafish. Dev Dyn. 1995;203: 253-310.

24. Cheng JC, Miller AL, Webb SE. Organization and function of microfilaments during late epiboly in zebrafish embryos. Dev Dyn. 2004;231:313-323

25. Cheng J, Fernando KAS, Veca LM, et al. Reversible accumulation of PEGylated single-walled carbon nanotubes in the mammalian nucleus. ACS Nano. 2008;2:2085-2094.
26. Kostarelos K. The long and short of carbon nanotube toxicity. Nat Biotechnol. 2008;26:774-776.

27. Poland CA, Duffin R, Kinloch I, et al. Carbon nanotubes introduced into the abdominal cavity of mice show asbestos-like pathogenicity in a pilot study. Nat Nanotechnol. 2008;3:423-428.

28. Williams D. The risks of nanotechnology. Med Device Technol. 2005;16(6):9-10.

29. Service RF. Nanotoxicology. Nanotechnology grows up. Science. 2004;304:1732-1734.

30. Warheit DB, Borm PJ, Hennes C, Lademann J. Testing strategies to establish the safety of nanomaterials: conclusions of an ECETOC workshop. Inhal Toxicol. 2007;19:631-643.

31. Fischer HC, Chan WCW. Nanotoxicity: the growing need for in vivo study. Curr Opin Biotechnol. 2007;18:565-571.

32. Garnett MC, Kallinteri P. Nanomedicines and nanotoxicology: some physiological principles. Occup Med (Lond). 2006;56:307-311. 


\section{Supplementary figure}
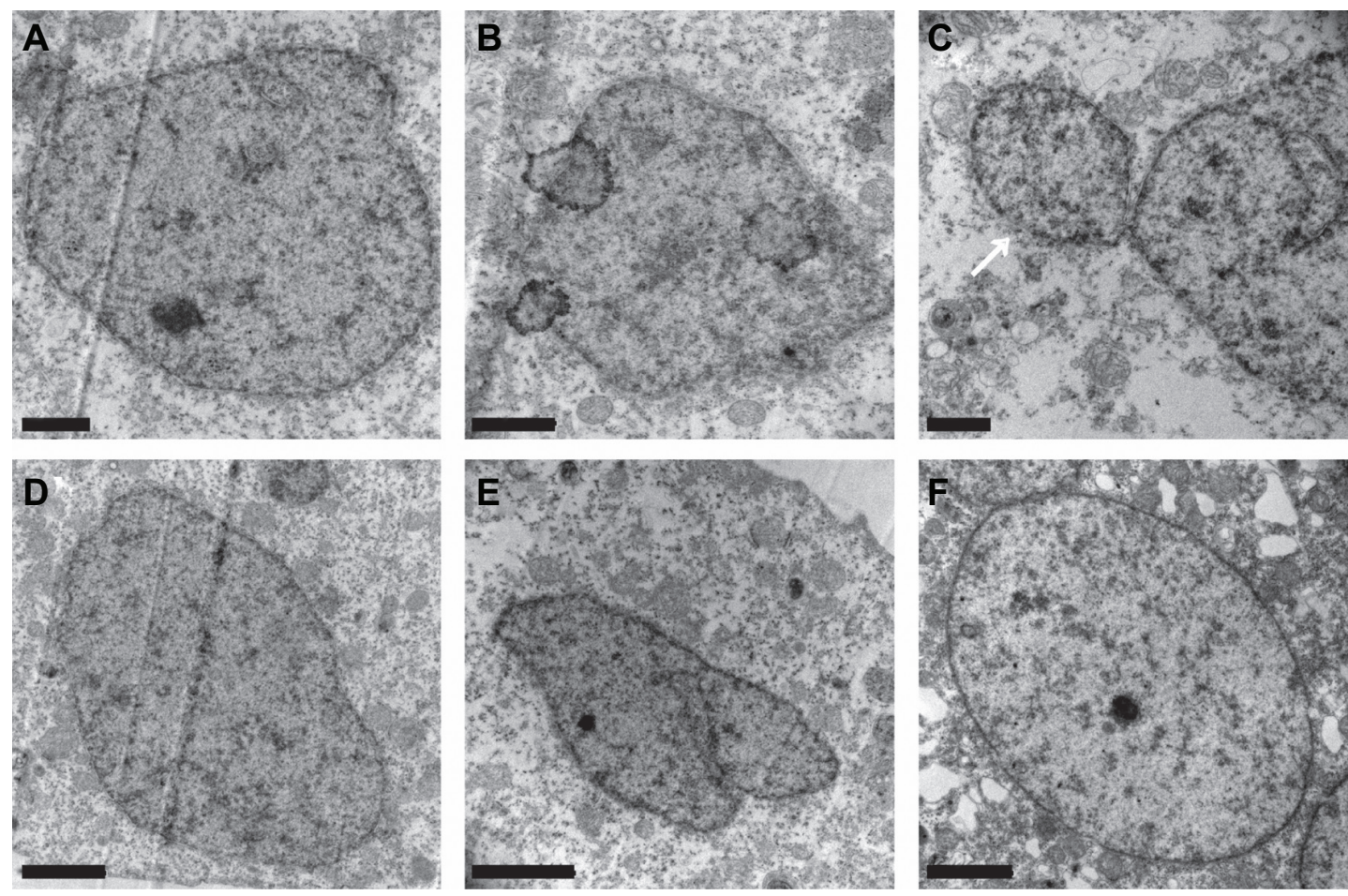

Figure SI Representative transmission electron microscopy graphs of the nucleus of the blastoderm cells of the embryos loaded with FITC-BSA-MWCNTs at one-cell stage (A-E) and untreated control (F) at 6 hpf. Embryos loaded with FITC-BSA-MWCNTs had irregular shape of nucleus and some micro-nucleus structure also appeared in treated embryos (see white arrow in C), while cells of the untreated embryos had regular round or a horse-shoe shaped nucleus at 6 hpf. Scale bar is I $\mu$ m in A, B and $C$, and $2 \mu \mathrm{m}$ in $\mathrm{D}, \mathrm{E}$ and $\mathrm{F}$.

Abbreviations: FITC-BSA-MWCNT, fluorescein isothiocyanate-labeled bovine serum albumin-functionalized multiwalled carbon nanotube; hpf, hours postfertilization.

International Journal of Nanomedicine

\section{Publish your work in this journal}

The International Journal of Nanomedicine is an international, peerreviewed journal focusing on the application of nanotechnology in diagnostics, therapeutics, and drug delivery systems throughou the biomedical field. This journal is indexed on PubMed Central, MedLine, CAS, SciSearch ${ }^{\circledR}$, Current Contents ${ }^{\circledR} /$ Clinical Medicine,

\section{Dovepress}

Journal Citation Reports/Science Edition, EMBase, Scopus and the Elsevier Bibliographic databases. The manuscript management system is completely online and includes a very quick and fair peer-review system, which is all easy to use. Visit http://www.dovepress.com/ testimonials.php to read real quotes from published authors. 